\title{
CiUdad es estilo: UnA definición estética DE LO URBANO
}

City is style: An aesthetic definition of the urban

\section{Adriana Duarte Romero \\ Benemérita Universidad Autónoma de Puebla, México ad.duarter@gmail.com}

RESUMEN: En el presente artículo de investigación teórica se aborda lo urbano desde una perspectiva peculiar sobre el tema: desde una perspectiva estética, es decir, como un estilo que adquiere su forma en las acciones cotidianas. Partiendo de las propuestas teóricas de autores como Georg Simmel y Michel Maffesoli sobre estética social, y sobre la vida cotidiana en la ciudad de Henri Lefebvre, planteo que la perspectiva del estilo abre la puerta a consideraciones críticas sobre la ciudad, y desde el ejemplo de la vida cotidiana en la ciudad reflexiono sobre la multiplicidad de la experiencia en relación con lo fragmentado de la vida urbana. Las relaciones entre el cuerpo y el espacio atan al estilo con la vida urbana y se convierten en formas en que habitamos la ciudad. A través del estilo urbano que se manifiesta en la vida cotidiana se entretejen experiencias corporales, subjetivas y espaciales que conectan e implican al habitante con su ciudad y a la ciudad con sus habitantes, y a su vez constituyen el origen de su crítica inmanente.

Palabras clave: estilo urbano, estética, ciudad, Georg Simmel, Henri Lefebvre.

RESUM: En el present article d'investigació teòrica s'aborda l'urbà des d'una perspectiva peculiar sobre el tema: des d'una perspectiva estètica, és a dir, com un estil que adquireix la seua forma en les accions quotidianes. Partint de les propostes teòriques d'autors com Georg Simmel i Michel Maffesoli sobre estètica social, i sobre la vida quotidiana a la ciutat de Henri Lefebvre, plantege que la perspectiva de l'estil obri la porta a consideracions crítiques sobre la ciutat, i des de l'exemple de la vida quotidiana a la ciutat reflexione sobre la multiplicitat de l'experiència en relació amb el fragmentat de la vida 
urbana. Les relacions entre el cos i l'espai lliguen a l'estil amb la vida urbana $i$ es converteixen en formes en què habitem la ciutat. A través de l'estil urbà que es manifesta en la vida quotidiana s'entretixen experiències corporals, subjectives i espacials que connecten $i$ impliquen l'habitant amb la seua ciutat i a la ciutat amb els seus habitants, i al seu torn constitueixen l'origen de la seua crítica immanent.

Paraules clau: estil urbà, estètica, ciutat, Georg Simmel, Henri Lefebvre.

\section{ABSTRACT:}

This theoretical research article approaches the urban from the particular perspective of aesthetics, that is, as a style that acquires its form in everyday actions. Starting from the theoretical proposals of Georg Simmel and Michel Maffesoli on social aesthetics, and Henri Lefebvre's work on everyday life in the city, I propose that the perspective of style opens the door to critical considerations of the city, and from the example of everyday life in the city I reflect on the multiplicity of experience in relation to the fragmented nature of urban life. The relationships between the body and space link style with urban life and become the ways that we inhabit the city. Through the urban style portrayed in everyday life, bodily, subjective and spatial experiences are woven together, connecting and involving the inhabitant with their city and the city with its inhabitants, and at the same time constitute the origin of its immanent critique.

KeYWORDs: urban style, aesthetics, city, Georg Simmel, Henri Lefebvre.

\section{Introducción}

L a ciudad y lo urbano han sido temas recurrentes en las ciencias sociales desde abordajes teóricos y metodológicos muy variados. Como característica de la modernidad, la ciudad constituye una espacio-temporalidad compleja que entreteje una urdimbre de fenómenos sociales, constantemente 
en cambio. El presente trabajo se enmarca dentro de las aportaciones interdisciplinares de la sociología de lo urbano, partiendo de la idea de que lo urbano construye y reconstruye a sus habitantes, al tiempo que es construido y reconstruido por ellos. Asimismo, se abordará el papel del estilo y la vida cotidiana en dichas construcciones y reconstrucciones.

Una búsqueda general deja claro que los estudios urbanos son inminentemente interdisciplinarios e implican acercamientos entre disciplinas como la sociología, la geografía humana, la antropología y la psicología social. Es posible encontrar trabajos que aborden lo urbano como el espacio contradictorio de la segregación y la desigualdad (Arellano \& Corona, 2018; Veiga, 1999; Vidal-Koppmann, 2007), como elemento central de la estructura económico-política del neoliberalismo (Capel, 2016) o como parte del proceso de subjetivación (Deciga \& Juárez-Salazar, 2018; Hidalgo, Alvarado, Santana, \& Paulsen, 2018).

Si bien es posible encontrar estudios urbanos que planteen la importancia de la vida cotidiana en la ciudad, la estética no suele ser parte del análisis, ya que con frecuencia esta se asocia únicamente al estudio de la belleza y la historia del arte. Abordar el tema de la ciudad desde la perspectiva estética desvanece la antigua distinción entre lo material y lo social. Se vuelve evidente que ambos procesos se alteran mutuamente en el flujo de lo cotidiano, dentro de un juego de apariencias «banales» que le dan forma y estructura a través de sus repeticiones cotidianas. Su papel en las prácticas de los citadinos crea un ambiente común que compone al estilo de vida urbano, como diferente de otros que lo rodean. La vida urbana no es algo que ocurra dentro de un lugar o en el interior de una envoltura, sino que incluye a la urbe misma y tiene movimiento, al igual que sus habitantes.

Es así que lo urbano tiene ciertas características esencialmente estéticas: el predominio de la apariencia, la intersección entre lo general y lo particular, la centralidad de lo sensorial, la teatralidad. De este modo, la apariencia en y de los espacios públicos se eslabona a la apariencia de las personas, cuya conjunción conforma el estilo urbano.

Georg Simmel llamó estética sociológica a sus análisis de las formas sociales como patrones de la vida social y cultural. Asimismo, se interesaba en 
los efectos sensoriales y mentales que tiene la vida moderna sobre los cuerpos y las conciencias de los individuos. Es decir, la estética se pone al servicio de la sociología, poniendo en primer plano aquellas formas que determinan la experiencia (Simmel, 1968; 2005). En este artículo parto de la misma estética sociológica que inició Simmel para interrogar a la ciudad y lo urbano desde la estética, rescatando aquellos elementos de la vida urbana que suelen quedar fuera de los análisis sociales, como la corporalidad y la repetitividad de la vida cotidiana. Así, me propongo destacar el papel que tiene el estilo no solo en la creación de la ciudad y la vida urbana, sino también en su crítica y su transformación.

\section{La vida urbana y la ciudad}

Aunque pareciera que la ciudad y lo urbano refieren más o menos a lo mismo, existen ciertas diferencias. Por ejemplo, algunos autores se refieren a los espacios urbanos como fracciones más accesibles de la ciudad, mientras que otros los identifican como la característica que la define. Para clarificar la diferencia entre ambos conceptos, partimos de una contraposición de posturas: en primer lugar, la de Henri Lefebvre, quien elabora una distinción teórica entre la ciudad y lo urbano, en la que considera a la ciudad como un sitio, un lugar donde se edifica la infraestructura compleja que alberga una población numerosa de personas que probablemente no se conocen entre ellas. Por otro lado, lo urbano constituye las prácticas que recorren y llenan la ciudad, los habitantes se mueven y son movidos por y para ello (Lefebvre, 1969).

Por otro lado, en su ensayo El animal público, Manuel Delgado dedica una sección entera a la discusión de lo urbano contra la urbe misma. Para él, la ciudad es «una composición espacial definida por la alta densidad poblacional y el asentamiento de un amplio conjunto de construcciones estables, una colonia humana densa y heterogénea conformada esencialmente por extraños entre sí». Lo urbano, por otro lado, se refiere a «un estilo de vida marcado por la proliferación de urdimbres relacionales deslocalizadas y precarias. Se entiende por urbanización, a su vez, "ese proceso consistente en 
integrar crecientemente la movilidad espacial en la vida cotidiana, hasta un punto en que esta queda vertebrada por aquella"» (Delgado, 1999, p. 23).

Desde esta última perspectiva, se entiende que la urbe es el escenario, el telón de fondo en el que el proceso de urbanización se encarga de dinamizar un ambiente significativo para quienes lo habitan. Vinculando así, a través de su experiencia, la serie de elementos desarticulados que componen la ciudad. El punto clave entonces no sería simplemente un habitante de la ciudad, sino un practicante de lo urbano. La ciudad sería el cuerpo y lo urbano, el alma; ya que es esta quien da las condiciones para el movimiento y la unión, pero a fin de cuentas son dos cosas separadas.

Parece claro que no toda la ciudad es urbana, a pesar de que todo lo urbano ocurre en la ciudad. Esta premisa, a simple vista acertada y hasta evidente, solo es sostenible si se construye desde una concepción en que la ciudad es de piedra, que las calles y los edificios, los espacios son inmóviles e inmutables y que la densidad demográfica y las denominaciones político-administrativas son la clave de su existencia. En ese caso, la ciudad sería la que se denomine como tal, sin tener en cuenta los procesos y fenómenos sociales que ocurren dentro de ella.

Si partimos de que el practicante de lo urbano es el agente estructurador de los fragmentos de la ciudad, tal parece que se trata un sujeto que se mantiene en aletargado, esperando ser despertado por algún evento inusual. En palabras de Delgado, se trata de actores que representan papeles intrascendentes en espera de su gran oportunidad: la indiferencia oculta en sus profundidades la potencia de la actividad, pero a fin de cuentas es indiferencia ${ }^{1}$. Cabría preguntarse si un sujeto de tales características sería realmente capaz de producir y mantener el proceso de urbanización, o si este proceso se detiene una vez que los sujetos lo abandonan para permanecer en las rutinas propias de la vida cotidiana. Una definición de la ciudad únicamente como lo material y de lo urbano como lo social ignora el hecho de que hay materialidad en lo social tanto como hay socialidad en lo material.

1. Otra lectura de la realidad social como un teatro en el que las personas fingen el papel de actores es la de Erving Goffman (2001) en su libro La presentación de la persona en la vida cotidiana. 
Volviendo a Lefebvre, la forma urbana ${ }^{2}$ se entiende como una «simultaneidad de acontecimientos, de percepciones, de elementos de un conjunto en lo real» (1969, p. 111), así, la forma urbana constituye un atributo de lo urbano en el que la multiplicidad de acontecimientos, situaciones e interacciones se construye y adquiere sentido a través de la vida cotidiana de las personas que comparten a la ciudad como su hogar.

Zenda Liendivit (2009) propone que el ambiente urbano es precisamente el que discurre en la relación e interacción entre la realidad psicológica y la realidad física de la ciudad. Es decir, que tanto lo psicosocial como lo material se presentan en ambos conceptos y aluden a la intersección entre el componente físico y el social de la ciudad y de la vida citadina. Es decir, que lo social y lo material no transcurren como realidades paralelas de carácter dualista, sino que están interconectadas.

En otras palabras, es imposible hablar de una ciudad compuesta solamente de elementos físicos o de una urbanidad que representa lo social y que articula lo fragmentado. Se entiende que lo urbano es parte de la ciudad tanto como ella de lo urbano, ya que el entramado material, social y afectivo es inseparable de cada uno de los elementos que lo rodean, y por lo tanto no puede entenderse sin ellos.

Como señala David Harvey, cualquier teoría que se detiene en lo unidimensional falla al pasar por alto la riqueza y complejidad de la experiencia:

Aprendemos a entender la ciudad desde múltiples perspectivas. Ella es, por un lado un laberinto incomprensible de cualidades caleidoscópicas: se gira el caleidoscopio, y vemos coloraciones y composiciones innumerables de la escena urbana. Aun así hay puntos nodales persistentes alrededor de los cuales la imagen de la ciudad confluye en algo más permanente y sólido. (2003, p. 42)

Lo urbano y la ciudad son, al mismo tiempo, lo permanente y lo transitorio, lo físico y lo espiritual, lo inusual y lo cotidiano. La ciudad deja de entenderse como el conjunto de espacios físicos desarticulados para convertirse en el escenario de la vida social urbana. Pero no en los términos de

2. Henri Lefebvre usa la categoría forma que describe Karl Marx en el primer tomo de El Capital, como un tipo particular de producción social e histórica. 
un fondo que solamente enmarca a los actores: al contrario, es un escenario que-late-junto-con sus protagonistas, y que determina en gran medida sus guiones, personajes y actuaciones, pero sin los cuales no existiría una ovación al terminar la obra.

Más aún, la ciudad y lo urbano son el punto de unión entre el tiempo largo y el tiempo corto, entre lo permanente y lo transitorio, entre lo móvil y lo inmóvil. Es en esas intersecciones donde reside su estética: se construyen y se reconstruyen en cada una de las pequeñas acciones que ocurren en el interior de la ciudad, lo que la convierte en un ente que, aunque cambiante, siempre parece ser el mismo, ya que siempre tiene la misma forma de ser, el mismo estilo.

\section{El estilo urbano}

Una característica indiscutible de las ciudades es que están en perpetuo movimiento, da la impresión de que no descansan. Quienes viven en una ciudad suelen disfrutar de la quietud o la lentitud de la no-ciudad en sus vacaciones o salidas de fin de semana.

La noción de ciudad en movimiento se relaciona más con personas que con objetos inanimados: toma su forma con lo que ocurre en las calles. Desde el siglo XVIII este proceso formativo se entiende como análogo al de un ser vivo compuesto por varias partes en las que el dinamismo es sinónimo de vida. Las calles y avenidas se asemejan al sistema circulatorio por sus flujos interminables de automóviles y de peatones, cuyo libre movimiento es inseparable de la ciudad misma. Los grandes parques tendrían una función equivalente a la de los pulmones, ya que al igual que estos, se encargan de oxigenar y purificar el ambiente (Sennett, 1997). La ciudad, entonces, sería un sistema complejo en el que todas sus partes deben funcionar en armonía, de lo contrario, colapsaría.

Honré de Balzac fue más allá al otorgarle características humanas a su ciudad:

En París hay ciertas calles que están en tanto desprestigio como puede estarlo cualquier hombre marcado con la infamia. También hay calles nobles, 
y calles que son simplemente decentes, y, por decirlo de alguna manera, calles adolescentes sobre las que el público aún no se forma una opinión. Hay calles asesinas; calles envejecidas; calles respetables; calles que siempre están limpias, calles que siempre están sucias; calles de industria mercantil y clase trabajadora. En pocas palabras, las calles de París tienen cualidades humanas y una fisionomía tal que nos deja con las impresiones contra las que no podemos poner resistencia. (Balzac, H. cit. en Harvey, 2003)

Este es otro tipo de analogía con los seres humanos. La primera, morfológica, encuentra similitudes físicas y funcionales en los espacios que le dan forma. Mientras que la segunda, avalada por la libertad poética, le concede a la ciudad atributos de personalidad, un genio y un estado de ánimo que además puede ir cambiando entre zonas y momentos. Las ideas de la ciudad que tiene dinamismo han aparecido una y otra vez, quizás denotando la necesidad de teorizar en ella lo etéreo y no solamente lo sólido de su configuración.

Así, aunque la dimensión física y geográfica es una parte importante, hace falta ver también el movimiento que entrañan las relaciones que cotidianamente tejen sus habitantes, las cuales dan sentido a la existencia de la ciudad en oposición a tipos de lugares. Ella es distinta a la provincia o al campo en muchos aspectos más que el de la apariencia: el ritmo y el movimiento son elementos que diferencian la ciudad de la no-ciudad.

La ciudad vive y se vive a un ritmo vertiginoso, debe hacerlo. El filósofo francés Charles Fourier incluso temía que una urbe monótona constituía veneno puro para la raza humana. Además, implica un ambiente en el que se armoniza la gran mayoría sus elementos que la representan. Es decir que hasta el más mínimo detalle, al ser analizado de forma aislada, remitirá de nuevo a ella. La prisa con la que andan los ejecutivos por las mañanas, las fachadas de las casas, las multitudes que se forman espontáneamente, los parques para hacer ejercicio los domingos: todo aquello que, aunque sea separado del contexto, sigue teniendo un «qué sé yo» que es totalmente urbano. Por supuesto, hay otros elementos que, si se separan del contexto, no se puede saber con certeza si son parte suya o de otra cosa, a pesar de que pertenezcan a una zona geográfica que formalmente recibe este nombre. A partir de esto podemos concluir que la ciudad no se cualifica inmediatamente con la denominación (geográfica o administrativa), sino que se trata de una cualidad más difusa 
y esquiva. Es decir, si bien necesita de una organización específica y de una delimitación geográfica, esto no es suficiente. En todo caso, son las condiciones que permiten y promueven la vida urbana, la acción social y la unidad simbólica colectiva.

Así las cosas, no todas las que reciben ese nombre son, en este sentido, ciudades. Al menos no en su totalidad. El mismo desarrollo urbano es un proceso finito para cada una: es imposible que crezca tanto hasta convertirse en una sola mancha en toda Europa, por ejemplo. Tal vez este sería un proyecto político y económico interesante, pero allí nunca se llegaría a desarrollar el estilo urbano como tal, por la falta de dos ingredientes claves: la acción social que solo un entorno de límites accesibles puede engendrar.

De forma que no toda la ciudad es ciudad, y no toda ciudad es la ciudad. Existe una gran diferencia entre la denominación política y la delimitación física, y la vida urbana que es recorrida, sentida y experimentada por sus habitantes; en pocas palabras, la que se vive. Por ello, no son suficientes los parámetros de densidad de población, alcanzar cierta cantidad de edificios corporativos o la inauguración del metro para considerar que cierto lugar es una ciudad en el sentido social del término.

El estilo urbano tiene una forma única, que la distingue de otras que pueden ser similares pero que no son lo mismo. De hecho, lo urbano requiere de otro tipo de espacios contrastantes para entenderse como tal. Al igual que una figura gestáltica necesita del fondo para delimitarse: no existiría sin sus contrapartes, si fuera imposible compararla con lo rural, la provincia, las playas, los campos y los desiertos. De esta forma, la existencia de la ciudad, de su ambiente urbano y su estilo de vida característico implica también la existencia de todos los demás tipos de espacios y estilos de vida presentes en otros lugares del mundo.

Lo urbano puede ser entendido como una forma, y más aún, como atmósfera. Contiene no solamente edificios, algunos parques y grandes cantidades de concreto y asfalto, sino también como aquella atmósfera que envuelve a las empresarias, empleadas, amas de casa y científicas sociales. Se entabla entonces una relación mutua, de cercanía tal, que sus habitantes constituyen también lo urbano: la ciudad forma parte de cada uno de ellos, así como los citadinos forman parte de ella. 
Ese ritmo común que tienen todos los aspectos de lo urbano es el estilo. «Estilo» significa marca, y como tal se imprime sobre los objetos que toca: en realidad toca a todo el ambiente urbano. Es por eso que en el estilo urbano es cuestión de compartir, se comparten gustos, gestos, colores y formas, ideas y modas (Fernández Christlieb, 2011). En otras palabras, se comparte la forma, el modo, la manera de vivir la ciudad.

En las urbes modernas la distinción entre lo dentro y lo fuera se difumina: el sujeto está en la ciudad tanto como ella está en el sujeto, el interior incluye al exterior. La modernidad permite esta diversificación de la vida privada dentro de la vida pública, las fronteras entre ambas son porosas y constantemente se entrecruzan.

Una interpretación similar fue propuesta años antes por Gaston Bachelard, para quien el hombre es un ser entreabierto. Inspirado por un fragmento de Donde se abrevan los lobos, de Tristan Tzara (en el cual la diferenciación dentro-fuera del personaje con su entorno espacial se vuelve muy borrosa), sostiene que «el cuarto es, en profundidad, nuestro cuarto, el cuarto está en nosotros. Ya no lo vemos» (Bachelard, 1975). En nuestro caso, hablamos de mucho más que un cuarto, hablamos de la casa que contiene al cuarto, de la colonia que contiene a la casa, y finalmente, de la ciudad que contiene todo lo anterior. Pero, de la misma forma en que el observador se funde con el cuarto en el que está ubicado, ambos se funden con su ciudad: la ciudad está en todo lo suyo y por lo tanto no es capaz de verla como un ente externo o independiente a su propia existencia. No es cuestión, pues, de continente y contenido, sino que ambas partes se con-funden, se encuentran concatenadas hasta la médula, y por lo tanto es imposible modificar una sin hacerlo también con la otra.

Ya decía Maffesoli: «las poéticas urbanas que se hacen posibles gracias a la familiaridad entre sus espacios y el observador, la naturalidad con la que ocurre y que no es exclusiva del sociólogo» (2007), pues todos, sin importar nuestros intereses y nuestra cantidad de reflexión al respecto, estamos imbuidos con ella.

Las características comunes entre la ciudad y sus habitantes derivan de los modos de vida, cuyo estilo urbano se vive en la cotidianidad y se reproduce, se materializa en sus cuerpos y en todos los espacios citadinos. Es así como 
se van insertando en la vida cotidiana, hasta parecer que ha existido siempre. Sin embargo, no es así, los estilos de vida citadinos parecen tener la misma naturaleza cambiante y efusiva de la moda: aquello que en algún momento fue la forma privilegiada de comportarse en público hoy nos parece gracioso o hasta ridículo. La ciudad se constituye como tal al ser un estilo, una forma, una manera.

Desde esta perspectiva, podemos ver al mismo fenómeno y concepto de lo urbano como un ente completo e indivisible, ya que su estilo no se puede fragmentar. De lo contrario dejaría de ser ese estilo en particular para convertirse en otro. La ciudad se rige siempre por el estilo que le da continuidad y que se comporta como una forma de equilibrio. La aparente fragmentación de la forma urbana es consecuencia de la multiplicidad rítmica, de situaciones y temporalidades, que ocurre simultáneamente cuando el estilo urbano se pone en práctica. El estilo urbano acoge una multiplicidad de experiencias, de ahí su apariencia fragmentaria.

A partir de estas consideraciones podemos hablar todo lo urbano a la vez, como todo lo que tenga su estilo propio. Tal estilo de vida implica ciertas formas de comportarse. Esto es precisamente lo que significa la «urbanidad», que pretendía enseñarse hace algunos años a través de manuales como el de Manuel Antonio Carreño, la cual, como el estilo urbano, puede imitarse a través de los modos de comportamiento. En este sentido, la urbanidad puede entenderse también como un arte y una manera: la de vivir con el otro en el mismo lugar.

La ciudad se fractura y se vuelve incomprensible si es vista como un cúmulo de elementos fragmentados y desarticulados que después se concatenan a través de múltiples experiencias subjetivas o mediante criterios solamente geográficos y políticos. Pero desde la perspectiva estética no puede fragmentarse. Es algo completo que nos trastoca a nosotros, los que la habitamos, convirtiéndonos en estetas cotidianos a través de nuestros movimientos y acciones dentro de su marco. 


\section{La estética urbana}

Según Aldo Rossi, en «los hechos urbanos hay algo que los hace muy semejantes, y no sólo metafóricamente, con la obra de arte; estos son una construcción en la materia, y a pesar de la materia; son algo diferente: son condicionados, pero también condicionantes» (2013, p. 12).

Alexander Baumgarten acuñó el término estética en el siglo XVIII retomando su origen etimológico, la raíz griega aisthesis, que se refiere a la región de la percepción y la sensación humana, en contraste con el dominio del pensamiento conceptual. Desde esta postura emergen planteamientos que, aunque son estéticos, no dejan de ser políticos. Las cuestiones de la vida sensorial, somática, la experiencia vivida, es decir, la cognición estética cobra importancia al entenderse como mediadora entre la generalidad de la razón y la particularidad de la sensación (Eagleton, 1990).

Comprender a la ciudad y lo urbano desde una perspectiva estética implica colocar el énfasis, encontrar la relación entre todas aquellas partes que constituyen una ciudad, sobre todo las que no son sólidas materialmente, pero que están presentes físicamente. Por un lado, incluye a los transeúntes preocupados por su rutina diaria; por otro, a quienes influyen de manera más evidente en su estructura. No existe, por lo tanto, un elemento que sea más urbano que los demás.

Comencemos retomando su analogía con un ente orgánico, la cual redimensiona el papel que juega en la vida de sus habitantes. Lejos de ser solamente el ambiente material que envuelve a los sujetos sin intervenir, manteniéndose al margen, recibiendo pasivamente las modificaciones que se acumulan sobre sus superficies; la ciudad participa, condiciona, cambia al unísono con los citadinos.

Tales características aparecen cuando se problematiza a la ciudad desde la estética, desde el estilo. Como señala Liendivit, se trata de un proceso que «está condicionado pero no estructurado o proyectado, no admite definiciones racionales y se lleva a cabo en la relación e interacción entre realidad psicológica y realidad física» (2009). La estética no está limitada por lo racional y es por eso que lo complementa. La atmósfera indescriptible que caracteriza al estilo urbano está cargada de aquellas mismas contradicciones, tensiones 
y antagonismos de la ciudad contemporánea, y aunque no se comprendan del todo, son sentidos por los habitantes de la ciudad.

La ciudad se hace con una forma de vivir y una forma de relacionarse, que dice Lewis Mumford, es al final una estética:

[...] en su sentido completo, entonces, es un plexo geográfico, una organización económica, un proceso institucional, un teatro de acción social y un símbolo estético de unidad colectiva. La ciudad fomenta arte y es arte; la ciudad crea al teatro y es el teatro. (Mumford, 1937, p. 93)

Lo urbano, entendido como estética, abre la perspectiva hacia aquellos elementos de interacción e intersubjetividad que la construyen en la vida cotidiana. Esto implica, para Maffesoli (2007), el arte que da estructura a la banalidad, es decir, que hace sociedad. En otras palabras, que se reconstruye con todos esos mínimos actos y lazos banales entre sus habitantes, creando estructuras de sentido que cimientan la vida social. Así, la vida urbana se va tejiendo con las experiencias cotidianas de las personas, con conversaciones casuales en la estación de autobuses, con reuniones de ex compañeros de la secundaria, con discusiones en la calle cuando ocurre un accidente vial. En resumen, vida urbana, arte y estética se sostienen bajo el mismo principio: con todas las pequeñas cosas que ocurren en su interior, sobre todo las que parecen ser más intrascendentes.

Se trata de una estética social que adquiere estructura a través de los siglos, desde las ciudadelas medievales, pasando por guerras y revoluciones, apropiaciones y expropiaciones del espacio público, automatizaciones de los medios de producción y creación de nuevos espacios de consumo ${ }^{3}$.

Así, el estilo urbano tiene más en común con el arte que con la lógica racional de lo objetivo. La convergencia de lo físico con lo subjetivo es llamada paradigma estético, entendido como la multiplicidad del yo y de su ambiente comunitario, la capacidad de sentir en común (Maffesoli, 2004). De nuevo, este sentimiento de comunidad se construye «banalmente» con las pequeñas cosas que a diario ocurren dentro de la ciudad; los viajes en el metro, las tertulias en un café o un bar, las filas para pagar o cobrar en el banco

3. Para más detalles sobre estos procesos, véase Mumford (1946). 
o los minutos de espera en la parada del autobús. Todas estas actividades cotidianas, aunque nebulosas, al unirse devienen la solidez del estilo citadino, y cada una de ellas está necesariamente situada espacial y temporalmente.

Podemos decir que la ciudad, al ser un modo, se vive como tal. Está presente en cada una de las acciones, movimientos y pensamientos de sus habitantes, que se en cuanto se construyen obtienen el aura urbana distintiva que se produce y se reproduce en la vida cotidiana.

\section{Vida cotidiana}

Un día cualquiera en la ciudad implica quizás despertarse temprano y salir a la calle, trasladarse durante un tiempo que puede ser corto o bastante largo (dependiendo de la distancia, el tráfico o las condiciones del camino), encontrarnos con otros, quizás saludar a algunos desconocidos, trabajar, comer, pasear, volver a casa y comenzar de nuevo al día siguiente. La vida cotidiana es rutinaria, y es también donde reside la estética. Todas y cada una de las actividades enumeradas más arriba implican la sensorialidad del cuerpo en su relación con la ciudad.

Para Georg Simmel, la ciudad excede sus límites geográficos de la misma forma que una persona excede su propio cuerpo: es ahí donde se expresa su existencia, pero es también apenas el inicio de su propio rango, que se extiende hasta la última consecuencia de sus actos tanto en el tiempo como en el espacio (Simmel, 2005).

Así, el estilo urbano, como característica inherente a la ciudad se construye y se reconstruye a cada momento. Nosotras, quienes la habitamos, transitamos por sus calles, poblamos sus edificios, trabajamos en sus oficinas y universidades, nos sentamos en sus parques y compramos en sus tiendas vamos creando los patrones de repetición característicos de la vida urbana: la velocidad se muestra como una característica que define la vida en la ciudad a diferencia de la vida en los espacios no-urbanos. Para Hartmut Rosa (2011), la aceleración de las transacciones económicas, el progreso tecnológico y el cambio cultural están asociados a la modernización y a la globalización. Sin embargo, no son procesos abstractos que solamente determinan las ciudades, 
sino que también son fenómenos psicosociales que se sienten y se viven en los cuerpos y en la vida cotidiana de los practicantes del estilo urbano.

A pesar del constante y vertiginoso movimiento urbano, nos parece que las ciudades son siempre las mismas, que los eventos se repiten una y otra vez sin muchas diferencias y que cada día será casi igual que el anterior. Esta repetición regular de eventos en tiempos y espacios determinados integran la vida cotidiana en la ciudad: los citadinos construimos y reconstruimos la ciudad a través de su estilo repetitivo, modo en el que también que somos construidos y reconstruidos por él.

La vida cotidiana es en sí misma contradictoria: puede ser reconfortante y frustrante a la vez, y es la estética la forma en la que logramos habitar esa contradicción cotidiana (Highmore, 2004). Así, podemos decir que habitamos al estilo urbano como un hogar, aquel que se construye con hábitos llenos de sentido práctico: la vida cotidiana y el estilo que esta erige no está vacío ni es homogéneo, se trata de un estilo múltiple, complejo y cercano a cada uno de los sujetos que participan en ellos.

La repetición como característica de la vida cotidiana permite apropiarnos de los espacios y reconocerlos como lugares familiares, porque los incidentes recurrentes son similares a los que hemos presenciado con anterioridad. En efecto, el espacio urbano se construye por cierto tipo de prácticas recurrentes combinadas con la permanencia de relaciones espaciales características. El tránsito por las calles es quizás uno de los ejemplos más claros de la estética de la vida cotidiana. Caminar por la ciudad es una situación corporeizada en la que el movimiento nos vuelve parte de la creación del estilo urbano. Cuando transitamos por los espacios — públicos y privados- que constituyen la ciudad, no somos solamente pasajeros: somos creadores de significados, de emociones, de percepciones, de afectos, a la vez que el espacio es creador de subjetividades.

Es así que en la vida cotidiana, tenemos la sensación de que las ciudades ya no son nuestras, de que el estilo urbano está siendo usurpado y controlado por una aceleración casi insostenible, o al menos de que la vida en la ciudad ya no es tan cómoda o satisfactoria como solía serlo o como imaginábamos que fuese. En la vida cotidiana se manifiestan las contradicciones de la forma urbana, así como sus tensiones y antagonismos, como crítica inmanente a 
la ciudad misma como producto del capitalismo tardío y sus procesos de modernización y globalización. La vida urbana aparece como fragmentada y sus habitantes como atomizados como consecuencia de estos procesos, sin embargo, es la misma vida cotidiana en la ciudad la que constituye el espacio ideal para su transformación, en cada una de sus repeticiones.

En palabras de Henri Lefebvre, esto sería ejercer nuestro derecho a la ciudad, que es necesariamente el derecho «a la vida urbana, a la centralidad renovada, a los lugares de encuentros y cambios, a los ritmos de vida y empleos del tiempo que permiten el uso pleno y entero de estos momentos y lugares» (Lefebvre, 1969). Es decir, el derecho a la ciudad implica la apropiación del estilo urbano, su realización a partir de criterios nuestros, cargados de sentido estético.

\section{Conclusiones}

Muchos de los aportes teóricos de las ciencias sociales se han centrado en el sentido racional de los fenómenos y procesos sociales, elaborando análisis sobre los símbolos y significados de cuestiones como la vida urbana. Sin embargo, los aportes de los teóricos de la sociología estética sugieren que el sentido de la vida urbana no solamente es racional, sino que también es estético, ya que a través del estilo urbano que se manifiesta en la vida cotidiana se entretejen múltiples experiencias corporales, subjetivas y espaciales que conectan e implican a los habitantes con su ciudad y a la ciudad con sus habitantes. Existe una estrecha relación corporal y sensorial entre ambos, a partir de la cual nos sentimos y somos parte del lugar que habitamos. Podemos hallar una especie de reflexión práctica o de sensación racional en los citadinos, con la cual se dan cuenta de las contradicciones de la vida urbana, su movimiento y su velocidad, o en pocas palabras: su estilo.

La vida cotidiana, con sus repeticiones aparentemente monótonas, constituye entonces la materia prima que sostiene la vida urbana en la ciudad. Asimismo, el estilo urbano es la envoltura del teatro de acción social y del símbolo estético de unidad colectiva que describimos antes con Lewis Mumford. Lo urbano se entreteje con la ciudad con el hilo de la vida coti- 
diana; y es que no se trata de dos procesos opuestos o separados, sino de dos partes complementarias que funcionan solamente en presencia de la otra. Así, la vida cotidiana es el reducto de aquel sentido práctico que se ejerce día a día: la particularidad de las experiencias cotidianas subjetivas en la ciudad se multiplica, se amplifica y se estructura en el estilo urbano, de modo que cada uno de sus habitantes participa en su creación.

Para Henri Lefebvre (2002) es necesaria una transformación radical de la vida cotidiana, en la que esta sea inundada por el arte. La estética, entonces, se convertiría en una herramienta de des-alienación. El estilo urbano (y la multiplicidad que implica) que se construye y reconstruye a diario en la ciudad puede ser en sí mismo una crítica práctica hacia la homogeneización de la vida y de la realidad. Es decir, que el estilo urbano y la estética misma serían campos de resistencia contra la depredación propia del capitalismo tardío y la modernización que le caracteriza. Partir de la noción estética del estilo para conceptualizar la ciudad y la vida urbana, como lo he hecho en este artículo, es una apuesta por repensar el aspecto político y radicalmente transformador de la vida cotidiana.

Desde esta perspectiva, caminar por las calles de la ciudad rumbo a la escuela o el trabajo o hacer las compras para la semana en el supermercado, no son acciones monótonas y vacías, son la esencia de nuestra relación con la ciudad. Las nociones expresadas en este trabajo no son sino una invitación a mirar lo estético y lo cotidiano desde otros ojos, aquellos llenos de esperanza en la transformación de la vida urbana, hacia una que sea mucho más satisfactoria para quienes hemos elegido a la ciudad como nuestro hogar. 


\section{Referencias}

Arellano, S. G., \& Corona, A. H. L. (2018). La forma urbana actual de las zonas metropolitanas en México: indicadores y dimensiones morfológicas. Estudios Demográficos y Urbanos, 34(1), 11-42.

Bachelard, G. (1975). La poética del espacio. México: Fondo de Cultura Económica.

Capel, H. (2016). La forma urbana en la ciudad postcapitalista. Biblio3W Revista Bibliográfica de Geografía y Ciencias Sociales, O(0). Recuperado de http://revistes.ub.edu/index.php/b3w/article/view/26366

Deciga, R. D., \& Juárez-Salazar, E. M. (2018). Ciudad adentro. Espacio, relato y extimidad en la escritura de la ciudad. URBS. Revista de Estudios Urbanos y Ciencias Sociales, 8(2), 73-84-84.

Delgado, M. (1999). El animal público. Hacia una antropología de los espacios urbanos. Barcelona: Anagrama.

Eagleton, T. (1990). The Ideology of the Aesthetic. Londres: Blackwell.

Fernández Christlieb, P. (2011). Lo que se siente pensar o la cultura como psicología. México: Taurus.

Goffman, E. (2001). La presentación de la persona en la vida cotidiana. Buenos Aires: Amorrortu.

Harvey, D. (2003). Paris, capital of modernity. Nueva York: Routledge.

Hidalgo, R., Alvarado, V., Santana, D., \& Paulsen, A. (2018). Metaespacio: la cáscara cosmopolita de un entorno inventado. Representaciones sobre el Barrio Italia, Santiago de Chile. Estudios Demográficos y Urbanos, 33(1), 79-110.

Highmore, B. (2004). Homework: Routine, social aesthetics and the ambiguity of everyday life. Cultural Studies, 18(2-3), 306-327.

Lefebvre, H. (1969). El derecho a la ciudad. Barcelona: Península.

Lefebvre, H. (2002). Critique of Everyday Life. Foundations of Sociology of the Everyday. Nueva York: Verso.

Liendivit, Z. (2009). La ciudad como problema estético. De la modernidad a la posmodernidad. Buenos Aires: Contratiempo.

Maffesoli, M. (2004). El tiempo de las tribus. El ocaso del individualismo en las sociedades posmodernas. México: Siglo XXI.

Maffesoli, M. (2007). En el crisol de las apariencias: Para una ética de la estética. México: Siglo XXI.

Mumford, L. (1937). What is a city? Architectural Record, 8(1). 
Mumford, L. (1946). The culture of cities. Londres: Secker \& Warburg.

Rosa, H. (2011). Aceleración social: consecuencias éticas y políticas de una sociedad de alta velocidad desincronizada. Persona y Sociedad, 25(1), 9-49.

Rossi, A. (2013). Arquitectura de la ciudad. México: Gustavo Gili.

SennetT, R. (1997). Carne y piedra. El cuerpo y la ciudad en la civilización occidental. Madrid: Alianza Editorial.

Simmel, G. (1968). Sociological aesthetics. En The Conflict in Modern Culture and Other Essays. Nueva York: Teachers College Press.

Simmel, G. (2005). La metrópolis y la vida mental. Bifurcaciones, 4(1).

Veiga, U. M. (1999). Pobreza, exclusión social y segregación espacial. Áreas. Revista Internacional de Ciencias Sociales, (19), 35-50.

Vidal-Koppmann, S. (2007). La expansión de la periferia metropolitana de Buenos Aires. Villas miseria y countries: de la ghettización a la integración de actores en el desarrollo local urbano. Scripta nova. Revista electrónica de geografía y ciencias sociales, 11(245). Recuperado de http://www.ub.es/geocrit/sn/sn-24542.htm 
